\title{
Optimum organ volume ranges for organs at risk dose in cervical cancer intracavitary brachytherapy
}

\author{
Zahra Siavashpour, MSc', Mahmoud Reza Aghamiri, PhD', Ramin Jaberi, PhD², Hamid Reza Dehghan Manshadi, MD³. \\ Reza Ghaderi, PhD!, Prof. Christian Kirisits, MSc, PhD ${ }^{4}$ \\ 'Department of Medical Radiation Engineering, Shahid Beheshti University, Tehran, Islamic Republic of Iran, ${ }^{2}$ Department of Radiotherapy \\ Cancer Institute, Tehran University of Medical Science, Tehran, Islamic Republic of Iran, ${ }^{3}$ Department of Radiotherapy, Hafte Tir Hospital, \\ Tehran, Islamic Republic of Iran, ${ }^{4}$ Department of Radiotherapy and Oncology. Comprehensive Cancer Center, Medical University of Vienna, \\ Austria
}

\begin{abstract}
Purpose: To analyze the optimum organ filling point for organs at risk (OARs) dose in cervical cancer high-doserate (HDR) brachytherapy.

Material and methods: In a retrospective study, 32 locally advanced cervical cancer patients (97 insertions) who were treated with 3D conformal external beam radiation therapy (EBRT) and concurrent chemotherapy during 20102013 were included. Rotterdam HDR tandem-ovoid applicators were used and computed tomography (CT) scanning was performed after each insertion. The OARs delineation and GEC-ESTRO-based clinical target volumes (CTVs) contouring was followed by 3D forward planning. Then, dose volume histogram (DVH) parameters of organs were recorded and patients were classified based on their OARs volumes, as well as their inserted tandem length.

Results: The absorbed dose to point A ranged between 6.5-7.5 Gy. $\mathrm{D}_{0.1 \mathrm{~cm}^{3}}$ and $\mathrm{D}_{2 \mathrm{~cm}^{3}}$ of the bladder significantly increased with the bladder volume enlargement $(p$ value $<0.05)$. By increasing the bladder volume up to about $140 \mathrm{~cm}^{3}$, the rectum dose was also increased. For the cases with bladder volumes higher than $140 \mathrm{~cm}^{3}$, the rectum dose decreased. For bladder volumes lower than $75 \mathrm{~cm}^{3}$, the sigmoid dose decreased; however, for bladder volumes higher than $75 \mathrm{~cm}^{3}$, the sigmoid dose increased. The $\mathrm{D}_{2 \mathrm{~cm}^{3}}$ of the bladder and rectum were higher for longer tandems than for shorter ones, respectively. The divergence of the obtained results for different tandem lengths became wider by the extension of the bladder volume. The rectum and sigmoid volume had a direct impact on increasing their $D_{0.1 \mathrm{~cm}^{3}}$ and $\mathrm{D}_{2 \mathrm{~cm}^{3}}$, as well as decreasing their $\mathrm{D}_{10}, \mathrm{D}_{30}$, and $\mathrm{D}_{50}$.

Conclusions: There is a relationship between the volumes of OARs and their received doses. Selecting a bladder with a volume of about $70 \mathrm{~cm}^{3}$ or less proved to be better with regards to the dose to the bladder, rectum, and sigmoid.

J Contemp Brachytherapy 2016; 8, 2: 135-142 DOI: $10.5114 /$ jcb.2016.59687
\end{abstract}

Key words: bladder volume, brachytherapy, cervical cancer, OARs.

\section{Purpose}

Twenty percent of deaths from cancer are due to gynecological (GYN) malignancies [1]. 3D image guided brachytherapy (IGBT) in combination with external beam radiotherapy (EBRT) is a favorite treatment option for all stages of cervical cancer, especially for inoperable patients $[2,3,4,5,6]$. To achieve sufficiently high-dose levels to the tumor, organs at risk (OARs) should be spared to reduce radiation related morbidity. Due to the dose-response effect, it is important to limit dose levels below defined dose constraints. The dose to the target and OARs can be optimized by using brachytherapy applicator with appropriate geometries and dwell time optimization based on the anatomical situation at the time of brachytherapy. The filling status of organs, like the bladder or rectum, has an effect on organ dosimetry [7]. In 2005 and 2006, GEC-ESTRO (Groupe Européen de Curiethérapie - European Society for Radiotherapy and Oncology) published recommendations on target definition and dose-volume histogram (DVH) parameters [8,9]. In 2012, the American Brachytherapy Society (ABS) endorsed the GEC-ESTRO recommendations to have "a common language" between distinctive brachytherapy centers $[10,11]$. The upcoming ICRU/GEC-ESTRO report 89 summarizes the state-of-the-art recommendation for reporting and recording dose and volume parameters [12]. Dose-volume histogram of OARs and clinical target volumes (CTVs), recommended by GEC-ESTRO, can be affected by bladder volume [13,14,15].

In the female pelvis, OARs are located in the close proximity to the intracavitary applicators, and there is a high-
Address for correspondence: Ramin Jaberi, PhD, Department of Radiotherapy, Cancer Institute, Tehran University of Medical Science, Tehran, Islamic Republic of Iran, phone: +98 912359 9974, fax: +98 218272 2329, $\bowtie$ e-mail: rjaberi@tums.ac.ir
Received: 26.01.2016

Accepted: 14.04.2016

Published: 27.04 .2016 
dose gradient from the dwell positions inside these applicators. Therefore, the influence of the bladder volume on the received dose by the OARs located in the pelvis can be evaluated to find the optimized bladder volume.

The effect of the bladder distention on received doses by the bladder, rectum, sigmoid, and small bowel during the HDR brachytherapy procedure has been discussed by previous scholars. Some of their research results were based on the doses to ICRU-38 points. Therefore in their studies despite the use of sectional images, some standard planes were chosen to deliver an acceptable dose to point A $[7,15]$. Several studies have been performed on a fixed protocol of bladder fillings but they could not find an optimum bladder volume to minimize the dose to OARs $[16,17]$.

The aim of this study is to find an optimum bladder volume to minimize the dose to pelvic OARs. Due to the lack of reproducibility in comparing previous research methods, this study was arranged as an extensive survey in four major HDR brachytherapy centers of Tehran, Iran, within more than two years research. In these centers, 3D forward treatment planning, manual optimizations, and dose reporting criteria were conducted based on the GEC-ESTRO recommendations for GYN brachytherapy, especially for the delineation of the intermediate and high risk-CTV $\left(\mathrm{CTV}_{\mathrm{IR}}\right.$ and $\mathrm{CTV}_{\mathrm{HR}}$, respectively) [5,6]. Then, effects of the bladder volume on the DVH parameters of the bladder, rectum, and sigmoid were assessed. The effect of tandem length on these parameters was also evaluated. Meanwhile, an optimum bladder distention was proposed for the bladder volume in GYN intracavitary brachytherapy, so that delivers the minimum dose to aforesaid normal tissues.

\section{Material and methods \\ Case selection}

A total of 32 patients (97 insertions) were selected from locally advanced cervical cancer patients treated in the four HDR brachytherapy centers from March 2010 to June 2013. Their median age was 56 years old (ranged between 47 and 64) and their median weight was $72 \mathrm{~kg}$ (ranged between 64 and 78). No exclusion was made either based on the patients' age or weight. The patients were treated with 3D conformal EBRT of 45-50 Gy for 5-6 weeks, with concurrent chemotherapy (50 mg cisplatin weekly). For all cases, the $\mathrm{CTV}_{\mathrm{HR}}$ was the cervix with no parametrical extension at time of brachytherapy. Therefore, all of these patients were candidates for intracavitary HDR tandem-ovoid brachytherapy without interstitial needles [18]. These patients were in different stages (IB2, IB, IIB) according to the FIGO staging [19]. The dose to point $\mathrm{A}$ of the studied cases was obtained between 6.5$7.5 \mathrm{~Gy}$.

\section{Applicator insertion}

In this study, Rotterdam HDR tandem-ovoid applicators (Nucletron, an Elekta company, Elekta AB, Stockholm, Sweden) with different tandem lengths (i.e., 3, 4, 5 , and $7 \mathrm{~cm}$ ) and ovoid caps of $2-3 \mathrm{~cm}$ diameter were used for different uterine and vaginal dimensions [20]. The cases were chosen from the patients treated using a 30-degree tilted tandem applicator, which is suitable for a wide range of anatomies. The main reason for this inclusion criterion was that in two of the study centers, the only available tandem angle was $30^{\circ}$ and in the other two, $93 \%$ of the cervical cancer patients were treated with this angle. By selecting these cases, one degree of freedom for statistical analysis was omitted. The insertions were performed under general anesthesia or partial sedation in the operation room.

\section{Patient preparation and imaging}

In all of the considered centers, a Foley catheter was inserted into the patients' bladder before implantations. Approximately $1 \mathrm{~cm}^{3}$ of Meglumin compound (Amp VISIPAQUE ${ }^{\mathrm{TM}} 320\left[50 \mathrm{~cm}^{3}\right]$, GE Healthcare, Ireland), as a contrast agent, was mixed with $6 \mathrm{~cm}^{3}$ normal saline and injected into the catheter balloon. In two centers, the bladder was filled with about $120 \mathrm{~cm}^{3}$ of normal saline solution and clamped right before the CT scanning. Therefore, scans were performed with a specified bladder volume. Then, the clip was opened and the bladder emptied passively (without help from a syringe) until the beginning of the treatment when the procedure was repeated and, then, the patient was treated by assuming the identical bladder filling. In the third center, the Foley catheter was kept open during the whole procedure (i.e., imaging and treatment). In the fourth center, the Foley catheter was completely clamped from the beginning to the end of the procedure. The time between the clamping and CT scanning was about 30 minutes, and the time between clamping and the dose delivery was approximately one hour. Positioning devices were used to guarantee the reproducibility of the patients positioning. In all of the patient preparation, scanning, and transferring steps, a flat backboard was used and the patients were fixed in the supine position, as they were during the time of the treatment.

Computed tomography scans of the whole pelvic were acquired using slices with a thickness of $3 \mathrm{~mm}$ (Somatom ${ }^{\circledR}$ DR, Siemens healthcare, Forchheim, Germany).

\section{Contouring, treatment planning, and treatment}

Patient's CT images were all imported to a 3D brachytherapy treatment planning system (Flexiplan ${ }^{\circledR}$, version 2.6, Isodose control, The Netherlands). The $\mathrm{CTV}_{\mathrm{HR}}$ and $\mathrm{CTV}_{\mathrm{IR}}$ of each patient were contoured by a radiation oncologist on the basis of GEC-ESTRO GYN working group recommendations and using the available MR images as guides for delineations (at the time of diagnosis and also before the brachytherapy) $[8,9]$. The bladder, rectum, and sigmoid were also contoured. The rectum was considered from the levator ani muscles until it was horizontally oriented in the axial slices. From there onwards, the sigmoid was considered to have started and was countered until the first part of the descending colon. As the study was a multicenter survey, the small bowel dose was not evaluated because of the inconsistency in its delineation. 
The brachytherapy prescribed dose per fraction was between 7-9 Gy to $D_{90}$ of the $\mathrm{CTV}_{\mathrm{HR}}$. This dose prescription was based on the linear quadratic model and EQD2 concept by considering the $\alpha / \beta$ of 10 Gy for the tumor and 3 Gy for late reacting normal tissues [21,22]. The treatment aims were to deliver $80-90 \mathrm{~Gy}$ as $\mathrm{D}_{90}$ of $\mathrm{CTV}_{\mathrm{HR}}$ (EQD2) and not more than 70-75 Gy to the rectum and sigmoid, and 80-90 Gy to the bladder $\left(\mathrm{D}_{2 \mathrm{~cm}^{3}}\right)$. These were the dose constrains permitted for the whole radiotherapy procedure (EBRT plus typically 3-4 fractions of brachytherapy). The planning aim for one individual fraction was, therefore, to reach an absorbed dose of around $7 \mathrm{~Gy}$. Inserted applicators were chosen from the software predefined library (reconstructions were done using three points). Treatment planning was initiated by rough dwell position/time suggestion of Flexiplan, followed by 3D adaption and manual optimization to ensure the desired planning aims were achieved (i.e., $\mathrm{D}_{90}$ of $\mathrm{CTV}_{\mathrm{HR}}$ was not less than 80-90 Gy and OARs doses were no greater than the dose constraints).

Flexitron ${ }^{\circledR}$ (Elekta AB, Stockholm, Sweden) was used as a HDR remote afterloading brachytherapy delivery system (Nucletron, an Elekta company, Elekta AB, Stockholm, Sweden), which was accompanied by ${ }^{192}$ Ir (Flexisource, Nucletron, an Elekta company, Elekta AB, Stockholm, Sweden) as an HDR stepping source.

\section{Classification}

The bladder, rectum, and sigmoid DVH parameters such as $\mathrm{D}_{0.1 \mathrm{~cm}^{3}}$ and $\mathrm{D}_{2 \mathrm{~cm}^{3}}$ were recorded in terms of percentages of the planning aim dose after each treatment plan. Patients were classified into four distinctive groups based on their bladder volumes (i.e., group A with less than $70 \mathrm{~cm}^{3}$, group B with 70 to $110 \mathrm{~cm}^{3}$, group $C$ with 110 to $170 \mathrm{~cm}^{3}$, and Group D with greater than $170 \mathrm{~cm}^{3}$ ). The rational for this type of patient classification was to have subgroups, in which the bladder volumes were the same as ones in the routine filling protocol of the clinics. Furthermore, another classification was made based on the physical tandem length and the patients were categorized into two groups (1) the patients who were treated with tandem lengths equal to or less than $4 \mathrm{~cm}$, and (2) those who were treated with tandem lengths longer than $4 \mathrm{~cm}$ (Table 1).

To consider the effect of patient rectum and sigmoid volumes on the DVH parameters of these organs, the volumes of these organs were also divided into different groups. The patients' rectal volumes were classified into $R_{1}\left(\leq 40 \mathrm{~cm}^{3}\right), R_{2}$ (between 40 and $\left.80 \mathrm{~cm}^{3}\right)$, and $R_{3}$ $\left(>80 \mathrm{~cm}^{3}\right)$. Also, the patients' sigmoid volume classification was as $S_{1}\left(\leq 35 \mathrm{~cm}^{3}\right)$ and $S_{2}\left(>35 \mathrm{~cm}^{3}\right)$ (Table 1$)$.

As mentioned earlier, two analysis steps were performed in order to find the approximate amount of the bladder volume, which caused the sigmoid and rectum to receive the minimum and maximum dose. In these analyses, we encountered some results that encouraged us to focus more on two out of the classified groups (i.e., B and $D)$. Therefore, these groups were further divided into the following subgroups; $B$ was divided into $B_{1}$ : 60 to $70 \mathrm{~cm}^{3}$, $\mathrm{B}_{2}: 70$ to $75 \mathrm{~cm}^{3}, \mathrm{~B}_{3}: 75$ to $90 \mathrm{~cm}^{3}$, and $\mathrm{B}_{4}: 90$ to $110 \mathrm{~cm}^{3}$.
Group $\mathrm{B}_{1}$ intentionally has an overlap with group $\mathrm{A}$, for finding the minimum bladder volume to deliver the minimum dose to the bladder more accurately. Group $\mathrm{D}$ was divided into $\mathrm{D}_{1}: 170$ to $180 \mathrm{~cm}^{3}, \mathrm{D}_{2}: 180$ to $200 \mathrm{~cm}^{3}, \mathrm{D}_{3}: 200$ to $220 \mathrm{~cm}^{3}$, and $\mathrm{D}_{4}>220 \mathrm{~cm}^{3}$, even though their statistical population was low. Note that the subgroups of B start from $60 \mathrm{~cm}^{3}$ in order to find the sigmoid minimum dose point more clearly.

To summarize the final results, we averaged the extracted DVH parameters of each classified groups (in terms of percentage of planning aim dose) and presented them below.

\section{Data analysis}

The DVHs of studied organs were compared descriptively among the cases in different classified groups to examine the effect of the bladder volume and tandem lengths on the OARs dose. In addition, we tried to find the optimum normal tissue volumes, which would lead to the minimum possible dose to the OARs. Numerical data analysis was performed through one-way ANOVA and general linear model (GLM)-univariate tests (confidence interval [CI] of 95\%) using SPSS (SPSS Statistics for Windows, Version 17.0. Chicago: SPSS Inc. USA).

To ensure the validity of the results and to prove the suitability of our data classification, normality of data distributions and homogeneity of their variance were tested by Kalmogorov-Smirnov (KS) and Levene tests, respectively. Considered continuous patients data included their FIGO stage, age, weight, and OARs' $\mathrm{D}_{2 \mathrm{~cm}^{3}}$ (as the maximum dose to the organs). Also the normality of the classified group was checked by choosing the classification criteria as the "Factor List" parameter in the SPSS.

\section{Results}

For the normality test, we checked that all KS tests passed significantly, i.e. sig. $>0.05$. Also, the normality distribution was checked graphically by the normal Q-Q and box plots, indicating that all normality distribution criteria passed (plots are not shown). The significance values of Levene tests were greater than 0.05 and tests for investigating the homogeneity of data variance passed.

\section{Bladder}

\section{The effect of bladder volume}

The results of this study indicate that the minimum dose to the most exposed small volumes of the bladder (i.e., $\mathrm{D}_{0.1 \mathrm{~cm}^{3}}$ and $\mathrm{D}_{2 \mathrm{~cm}^{3}}$ ), increased by the bladder volume enlargement. An example of this claim is Figure 1, which illustrates how the bladder wall closes off to the high

Table 1. Frequency of classified cases (\#)

\begin{tabular}{lccccccccc} 
Tandem & \multicolumn{4}{c}{ Bladder } & \multicolumn{4}{c}{ Rectum } & \multicolumn{3}{c}{ Sigmoid } \\
\cline { 2 - 10 } & length & B & C & D & $\mathrm{R}_{1}$ & $\mathrm{R}_{2}$ & $\mathrm{R}_{3}$ & $\mathrm{~S}_{1}$ & $\mathrm{~S}_{2}$ \\
\hline $\mathrm{I} \leq 4 \mathrm{~cm}$ & 18 & 13 & 8 & 10 & 20 & 22 & 7 & 35 & 14 \\
\hline $\mathrm{II}>4 \mathrm{~cm}$ & 16 & 8 & 15 & 9 & 12 & 28 & 8 & 27 & 21
\end{tabular}



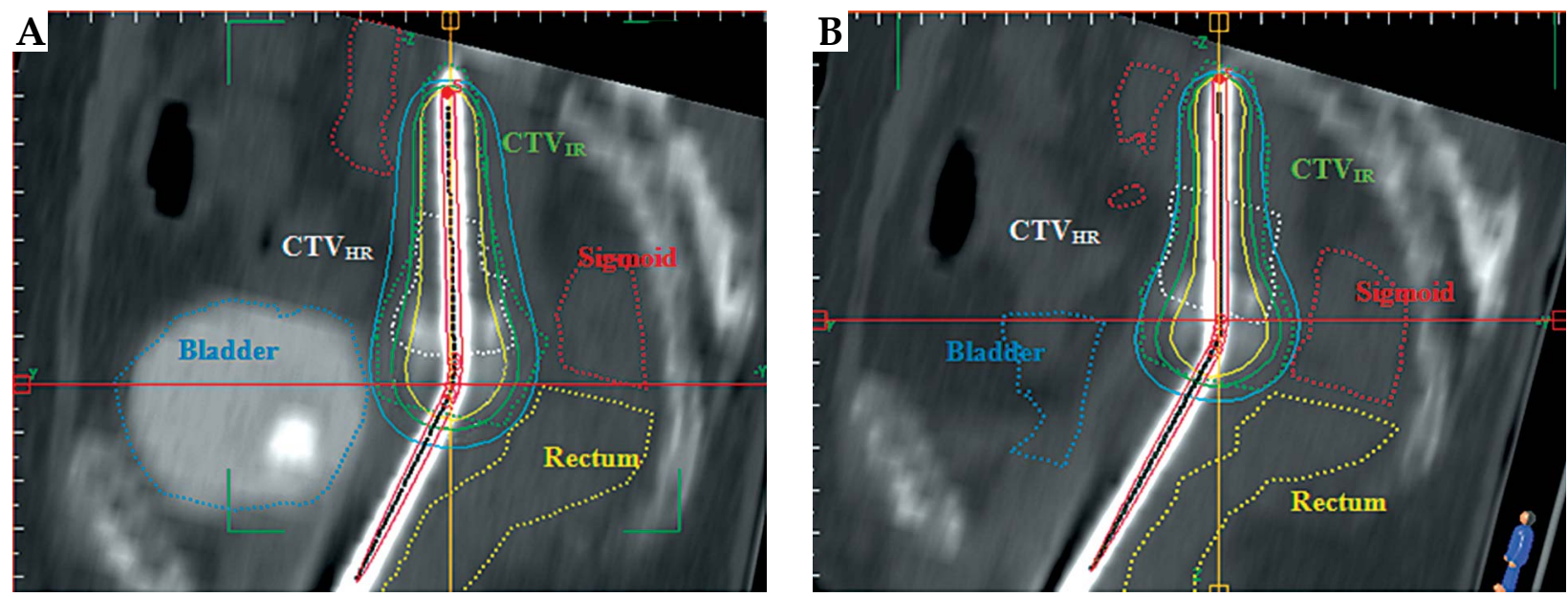

Fig. 1. An example of bladder volume effect on the bladder, rectum and sigmoid dose volume histogram (DVH) parameters: (A) bladder volume $=137.1 \mathrm{~cm}^{3}$, bladder $\mathrm{D}_{2 \mathrm{~cm}^{3}}=61.4 \%$, rectum $\mathrm{D}_{2 \mathrm{~cm}^{3}}=59.7 \%$, sigmoid $\mathrm{D}_{2 \mathrm{~cm}^{3}}=61.4 \% ;($ B $)$ bladder volume $=44.6 \mathrm{~cm}^{3}$, bladder $\mathrm{D}_{2 \mathrm{~cm}^{3}}=40.2 \%$, rectum $\mathrm{D}_{2 \mathrm{~cm}^{3}}=52.5 \%$, sigmoid $\mathrm{D}_{2 \mathrm{~cm}^{3}}=61.3 \%$ (\%: percentage of the planning aim dose)

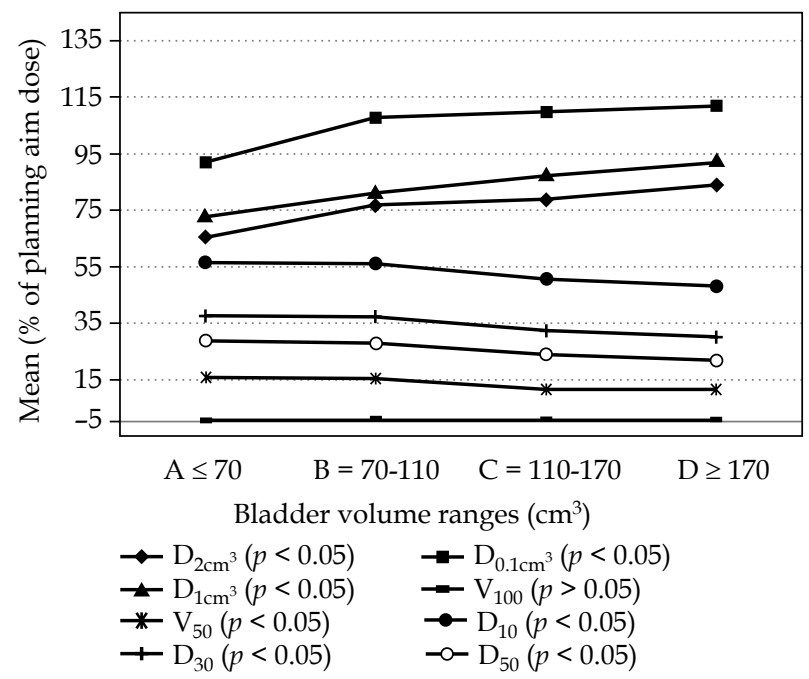

Fig. 2. Variation trend of bladder dose volume histogram (DVH) parameters versus the bladder volume

dose region by increasing its volume. On the other hand, large volume doses (i.e., $\mathrm{D}_{10}, \mathrm{D}_{30}$, and $\mathrm{D}_{50}$ ) decreased, although with a lower slope (Figure 2). For volume parameters (e.g., $\mathrm{V}_{100}$; the $\mathrm{V}_{100}$ is the volume, which receives the $\left.\mathrm{D}_{90}\right)$ there were no significant differences ( $p$ value $\left.>0.05\right)$ between different groups. However, the overall results indicated a reduction in irradiated bladder volumes with the increase in total bladder volume (Figure 2). In all the classified groups, except $\mathrm{V}_{100}$, the differences in bladder DVH parameters were statistically significant (e.g. $p$ value was obtained as 0.007 and 0.02 for $\mathrm{D}_{2 \mathrm{~cm}^{3}}$ and $\mathrm{D}_{0.1 \mathrm{~cm}^{3}}$, respectively).

\section{The effect of tandem length}

The statistical results of the effect of tandem lengths on the bladder dose tests were significant $(p$ values were 0.024 and 0.004 for $\mathrm{D}_{2 \mathrm{~cm}^{3}}$ and $\mathrm{D}_{10}$, respectively), except for $\mathrm{D}_{0.1 \mathrm{~cm}^{3}}$. The results show that the dose to bladder and the irradiated volume of bladder increased when longer tandems were used (Table 2). This increase in the dose was equal to $4.8 \%$ and $4.9 \%$ (\% of the planning aim dose) for $\mathrm{D}_{2 \mathrm{~cm}^{3}}$ and $\mathrm{D}_{10}$ of this organ, respectively.

\section{The effect of bladder volume and tandem length}

No statistically significant outcome on the bladder dose was obtained when considering applicator lengths and bladder volumes (GLM test), simultaneously.

\section{Rectum}

The effect of bladder volume on rectum dose

The rectum dose increased as the bladder volume increased up to about $140 \mathrm{~cm}^{3}$. For higher bladder volumes,

Table 2. Bladder and rectum dose volume histogram (DVH) parameters of two classified groups of tandem length

\begin{tabular}{|c|c|c|c|c|c|c|c|c|c|c|c|c|}
\hline \multirow[t]{3}{*}{ T.L (cm) } & \multicolumn{7}{|c|}{ Bladder DVH parameters } & \multicolumn{5}{|c|}{ Rectum DVH parameters } \\
\hline & \multicolumn{5}{|c|}{ Mean $\%$ of planning aim dose $\pm 1 \sigma$} & \multicolumn{2}{|c|}{$\begin{array}{c}\text { Mean } \% \\
\text { of volume } \pm 1 \sigma\end{array}$} & \multicolumn{5}{|c|}{ Mean\% of planning aim dose $\pm 1 \sigma$} \\
\hline & $\mathrm{D}_{2 \mathrm{~cm}^{3}}$ & $\mathrm{D}_{0.1 \mathrm{~cm}}$ & $\mathrm{D}_{10}$ & $\mathrm{D}_{30}$ & $\mathrm{D}_{50}$ & $V_{100}$ & $\mathrm{~V}_{50}$ & $\mathrm{D}_{2 \mathrm{~cm}}$ & $\mathrm{D}_{0.1 \mathrm{~cm}^{3}}$ & $\mathrm{D}_{10}$ & $\mathrm{D}_{30}$ & $\mathrm{D}_{50}$ \\
\hline$\leq 4$ & $\begin{array}{r}70.0 \\
\pm 17.5\end{array}$ & $\begin{array}{r}99.9 \\
\pm 29.0\end{array}$ & $\begin{array}{r}47.9 \\
\pm 13.1\end{array}$ & $\begin{array}{l}30.6 \\
\pm 9.3\end{array}$ & $\begin{array}{r}22.5 \\
\pm 7.2 \\
\end{array}$ & $\begin{array}{c}0.4 \\
\pm 0.6 \\
\end{array}$ & $\begin{array}{l}10.4 \\
\pm 8.1 \\
\end{array}$ & $\begin{array}{r}59.8 \\
\pm 9.6 \\
\end{array}$ & $\begin{array}{r}82.6 \\
\pm 13.6\end{array}$ & $\begin{array}{r}50.4 \\
\pm 10.0 \\
\end{array}$ & $\begin{array}{l}34.7 \\
\pm 7.3\end{array}$ & $\begin{array}{l}26.6 \\
\pm 5.8 \\
\end{array}$ \\
\hline$>4$ & $\begin{array}{r}74.8 \\
\pm 20.9\end{array}$ & $\begin{array}{l}104.0 \\
\pm 31.5\end{array}$ & $\begin{array}{r}52.8 \\
\pm 16.3\end{array}$ & $\begin{array}{r}35.8 \\
\pm 12.0\end{array}$ & $\begin{array}{r}27.3 \\
\pm 9.6\end{array}$ & $\begin{array}{c}0.7 \\
\pm 1.2\end{array}$ & $\begin{array}{r}14.9 \\
\pm 12.3\end{array}$ & $\begin{array}{l}65.3 \\
\pm 9.2\end{array}$ & $\begin{array}{r}87.9 \\
\pm 13.7\end{array}$ & $\begin{array}{l}54.6 \\
\pm 8.0\end{array}$ & $\begin{array}{l}38.7 \\
\pm 6.8\end{array}$ & $\begin{array}{l}30.3 \\
\pm 6.1\end{array}$ \\
\hline
\end{tabular}


the rectum dose showed a decreasing trend (Figure 3). However, the ANOVA test results were not significant. As it is illustrated in Figure 3, the dose to the rectum was maximum when the volume of the bladder was about $120-140 \mathrm{~cm}^{3}$. In this range, $\mathrm{D}_{0.1 \mathrm{~cm}^{3}}$ and $\mathrm{D}_{2 \mathrm{~cm}^{3}}$ of the rectum became about $90 \%$ and $69 \%$ of the planning aim dose, respectively. On the other hand, these rectum DVH parameters (i.e., $D_{0.1 \mathrm{~cm}^{3}}$ and $\mathrm{D}_{2 \mathrm{~cm}^{3}}$ ) became $82 \%$ and $60 \%$ when the bladder volume was below $70 \mathrm{~cm}^{3}$, and they were $84 \%$ and $62 \%$ when the bladder volume was more than $170 \mathrm{~cm}^{3}$, respectively (Figure 3).

\section{The effect of tandem length on rectum dose}

Like the bladder, the length of the tandem had a significant and direct influence on the rectum DVH parameters ( $p$ value of 0.004 and 0.026 for $\mathrm{D}_{2 \mathrm{~cm}^{3}}$ and $\mathrm{D}_{10}$, respectively). For treatments with longer tandem lengths, the rectum dose was higher (Table 2). For patients who were treated with longer tandem lengths $(>4 \mathrm{~cm}), \mathrm{D}_{2 \mathrm{~cm}^{3}}$ and $\mathrm{D}_{10}$ of the rectum were $5.5 \%$ and $4.2 \%$ (percentage of the planning aim dose) higher, respectively. Multivariate statistical test results showed that there were no statistically significant effects from tandem lengths on the rectal dose. In different subgroups of bladder volumes, the rectal dose was only about $4 \%$ higher for those treated with tandem lengths of $>4 \mathrm{~cm}$.

\section{The effect of rectum volume and tandem length on rectum dose}

As expected, by increasing the rectum volume, the $\mathrm{D}_{0.1 \mathrm{~cm}^{3}}$ and $\mathrm{D}_{2 \mathrm{~cm}^{3}}$ parameters increased; however, $\mathrm{D}_{10}, \mathrm{D}_{30}$, and $\mathrm{D}_{50}$ decreased (Figure 4 ). While the rectum volume increased, the effect of tandem lengths on the rectum's DVH parameters became more dominant; this can be seen in Figure 4 where the discrepancies of the two related diagrams intensify for more filled rectums. Rectum $\mathrm{D}_{0.1 \mathrm{~cm}^{3}}$ and $\mathrm{D}_{2 \mathrm{~cm}^{3}}$ discrepancies were about $3 \%$ (of the planning aim dose) for cases with rectum volumes of less than $40 \mathrm{~cm}^{3}$ and $10-13 \%$ for rectum volumes greater than $80 \mathrm{~cm}^{3}$.

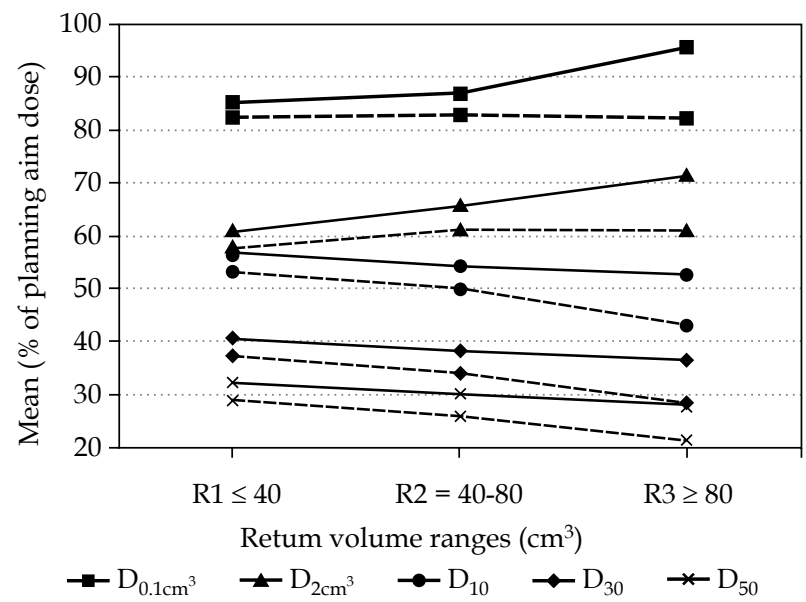

Fig. 4. Variation of rectum dose volume histogram (DVH) parameter versus the rectum volume and tandem length (dashed line indicates tandem length $\leq 4 \mathrm{~cm}$ and solid line indicates tandem length $>4 \mathrm{~cm}$ )

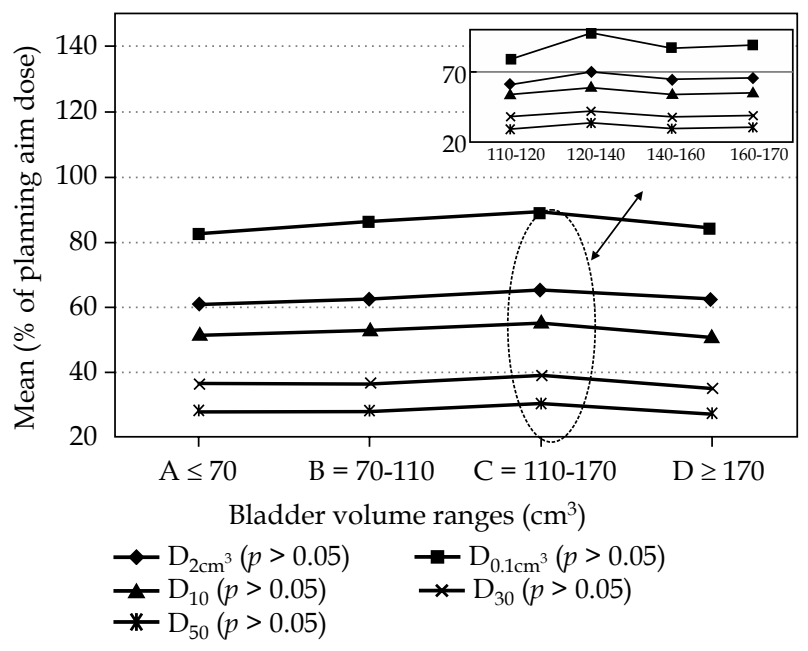

Fig. 3. Variation of rectum dose volume histogram (DVH) parameter versus the bladder volume

\section{Sigmoid}

The effect of bladder volume on sigmoid dose

Obtained results for the sigmoid are presented in Figures 1 and 5. The minimum dose to the sigmoid was obtained in the case of a bladder volume of 70 to $75 \mathrm{~cm}^{3}$. In this range, for example, $\mathrm{D}_{0.1 \mathrm{~cm}^{3}}$ and $\mathrm{D}_{2 \mathrm{~cm}^{3}}$ became $44 \%$ and $31 \%$ of the planning aim dose, respectively.

\section{The effect of tandem length on sigmoid dose}

As expected, tandem lengths had a direct influence on the sigmoid dose. The sigmoid received a significantly higher dose when longer tandems were used. For example, when the bladder volume was more than $170 \mathrm{~cm}^{3}$, the mean values of sigmoid $\mathrm{D}_{0.1 \mathrm{~cm}^{3}}$ and $\mathrm{D}_{2 \mathrm{~cm}^{3}}$ (in terms of the percentage of the planning aim dose) were $34 \%$ and $22 \%$ for patients with $\mathrm{T}>4 \mathrm{~cm}$ and $\mathrm{T} \leq 4 \mathrm{~cm}$, respectively. For those whose bladder volume was $70-110 \mathrm{~cm}^{3}$, these values were $17 \%$ and $11 \%$, respectively. However,

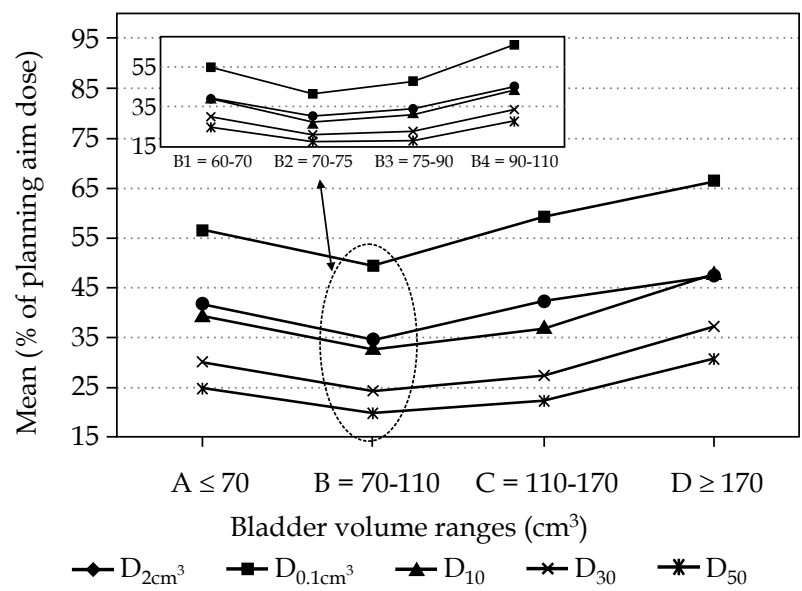

Fig. 5. Variation of sigmoid dose volume histogram (DVH) parameter versus the bladder volume 


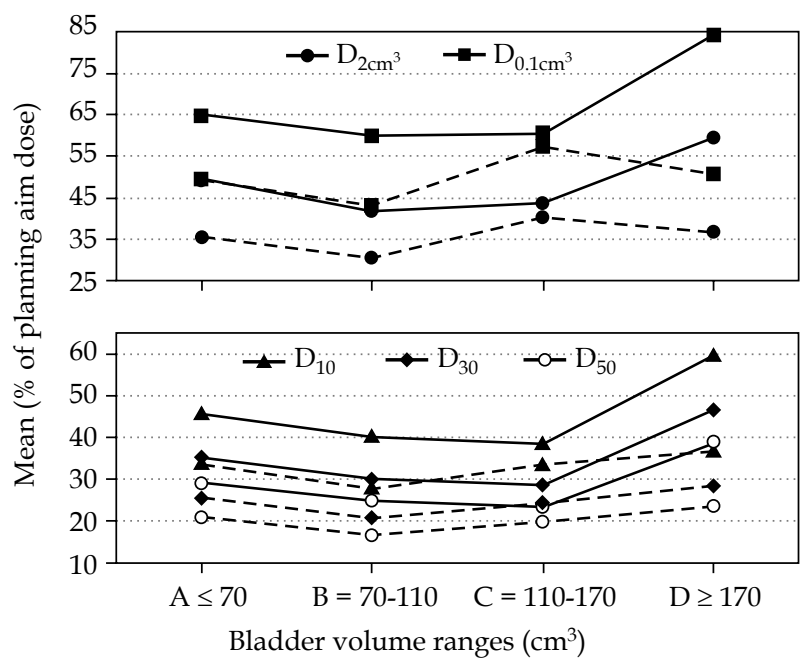

Fig. 6. Variation of sigmoid dose volume histogram (DVH) parameter versus the bladder volume and tandem length (dashed line indicates tandem length $\leq 4 \mathrm{~cm}$ and solid line indicates tandem length $>4 \mathrm{~cm}$ )

this difference in sigmoid dose was decreased when the volume of the bladder was $110-170 \mathrm{~cm}^{3}$. For example, the difference in sigmoid $D_{0.1 \mathrm{~cm}^{3}}$ and $\mathrm{D}_{2 \mathrm{~cm}^{3}}$ was only $\sim 3 \%$ in this particular range. Figure 6 illustrates that the effect of the bladder volume on received doses by the sigmoid are less pronounced for patients treated with shorter tandem lengths $(\leq 4 \mathrm{~cm})$ than the longer ones $(>4 \mathrm{~cm})$.

\section{The effect of sigmoid volume and tandem length on sigmoid dose}

By increasing the sigmoid volume, its minimum dose to the most exposed volumes (i.e., $\mathrm{D}_{0.1 \mathrm{~cm}^{3}}$ and $\mathrm{D}_{2 \mathrm{~cm}^{3}}$ ) increased to about $10 \%$ and $8 \%$ of the planning aim dose, respectively. However, the $\mathrm{D}_{10}, \mathrm{D}_{30}$, and $\mathrm{D}_{50}$ decreased as expected (Table 3). Multivariate statistical test results for simultaneous effects of sigmoid volume and tandem length on sigmoid dose were significant (Table 3). For example, $p$ values of this GLM test were 0.042 and 0.036 for $\mathrm{D}_{2 \mathrm{~cm}^{3}}$ and $\mathrm{D}_{10}$, respectively.

\section{Discussion}

The effects of bladder, rectum, and sigmoid volumes as well as the tandem length on DVH parameters of the said organs in HDR intracavitary brachytherapy of cervical cancer patients were assessed. Also, the optimum volume range of the bladder was searched so that within this range, the mentioned OARs received their minimum dose values. In a study previously conducted by the same authors, the effects of two bladder filling situations (full and empty) on the OARs dose of individual patients were compared. In this paper the analysis was focused to find the optimum organ volume range, which were achieved via analyzing the data of 97 brachytherapy cases [23].

The first reason for the mentioned patient classification was that different brachytherapy centers apply different bladder filling protocols; they typically inject 1 to 3 full $60 \mathrm{~cm}^{3}$ syringes of normal saline to the bladder Foley catheter before scanning it $[15,24,25]$. Another proto$\mathrm{col}$ for the centers, which believe in empty bladders is to leave the Foley clamp open $[10,20]$. However, according to our findings, even in opened Foley conditions, there would still be about $60-70 \mathrm{~cm}^{3}$ urine. Another reason for this type of classification was to reach an acceptable statistical population for each subgroup.

Our results for the evaluation of bladder dose variations by increasing the bladder volumes are in close consistency with previous studies (Figure 2) $[14,16,26]$. For example, $\mathrm{D}_{2 \mathrm{~cm}^{3}}$ of this organ increased from about $75 \%$ to $95 \%$ (of the planning aim dose) by increasing the bladder volume from less than 70 to more than $170 \mathrm{~cm}^{3}$. It is difficult to extrapolate our values for empty bladder filling status. In principle, the dose to the bladder should decrease further. On the other hand, for a completely empty bladder, the anterior bladder wall would be in contact with the posterior bladder wall and, thus, the total volume of irradiated bladder wall is increased. This effect is not directly visible with DVH analysis. Therefore, there is no evidence to treat patients with a completely empty bladder at this point. According to the results attained from the rectum, it can be concluded that the rectum dose can be affected by the bladder volume.

Table 3. Analysis results of sigmoid volume and tandem length effect on the sigmoid dose volume histogram (DVH) parameters

\begin{tabular}{|c|c|c|c|c|c|c|c|c|c|c|c|c|}
\hline \multirow[t]{2}{*}{$\begin{array}{l}\text { Sig. vol. } \\
\left(\mathrm{cm}^{3}\right)\end{array}$} & \multicolumn{5}{|c|}{$\begin{array}{c}\text { DVH parameters } \\
\text { (Mean\% of planning aim dose } \pm 1 \sigma \text { ) }\end{array}$} & \multirow[t]{2}{*}{$\begin{array}{l}\text { T. L } \\
(\mathrm{cm})\end{array}$} & \multirow[t]{2}{*}{$\begin{array}{l}\text { Sig. vol. } \\
\left(\mathrm{cm}^{3}\right)\end{array}$} & \multicolumn{5}{|c|}{$\begin{array}{c}\text { DVH parameters } \\
\text { (Mean\% of planning aim dose } \pm 1 \sigma \text { ) }\end{array}$} \\
\hline & $\mathrm{D}_{2 \mathrm{~cm}^{3}}$ & $\mathrm{D}_{0.1 \mathrm{~cm}^{3}}$ & $\mathrm{D}_{10}$ & $D_{30}$ & $D_{50}$ & & & $\mathrm{D}_{2 \mathrm{~cm}^{3}}$ & $\mathrm{D}_{0.1 \mathrm{~cm}^{3}}$ & $\mathrm{D}_{10}$ & $D_{30}$ & $\mathrm{D}_{50}$ \\
\hline \multirow[t]{2}{*}{$\leq 35$} & $\begin{array}{c}38.6 \pm \\
17.3\end{array}$ & $\begin{array}{c}54.1 \pm \\
26.9\end{array}$ & $\begin{array}{c}39.4 \pm \\
18.9\end{array}$ & $\begin{array}{c}30.7 \pm \\
14.5\end{array}$ & $\begin{array}{c}25.6 \pm \\
12.0\end{array}$ & $<4$ & $\leq 35$ & $\begin{array}{c}31.3 \pm \\
14.3\end{array}$ & $\begin{array}{c}43.7 \pm \\
21.9\end{array}$ & $\begin{array}{c}31.7 \pm \\
14.9\end{array}$ & $\begin{array}{c}24.6 \pm \\
11.0\end{array}$ & $\begin{array}{c}20.3 \pm \\
8.7\end{array}$ \\
\hline & & & & & & & $>35$ & $\begin{array}{c}44.3 \pm \\
13.8\end{array}$ & $\begin{array}{l}62.6 \pm \\
20.4\end{array}$ & $\begin{array}{c}35.8 \pm \\
11.1\end{array}$ & $\begin{array}{c}25.2 \pm \\
7.0\end{array}$ & $\begin{array}{c}19.8 \pm \\
5.4\end{array}$ \\
\hline \multirow[t]{2}{*}{$>35$} & $\begin{array}{c}46.4 \pm \\
14.4\end{array}$ & $\begin{array}{l}63.6 \pm \\
20.3\end{array}$ & $\begin{array}{c}38.1 \pm \\
11.8\end{array}$ & $\begin{array}{c}27.5 \pm \\
8.4\end{array}$ & $\begin{array}{c}21.9 \pm \\
6.6\end{array}$ & $>4$ & $\leq 35$ & $\begin{array}{c}48.1 \pm \\
16.4\end{array}$ & $\begin{array}{c}67.7 \pm \\
27.2\end{array}$ & $\begin{array}{c}49.4 \pm \\
19.2\end{array}$ & $\begin{array}{c}38.7 \pm \\
14.8\end{array}$ & $\begin{array}{c}32.1 \pm \\
11.7\end{array}$ \\
\hline & & & & & & & $>35$ & $\begin{array}{c}47.8 \pm \\
14.9\end{array}$ & $\begin{array}{l}64.3 \pm \\
20.6\end{array}$ & $\begin{array}{c}39.6 \pm \\
12.3\end{array}$ & $\begin{array}{c}29.0 \pm \\
9.0\end{array}$ & $\begin{array}{c}23.2 \pm \\
7.1\end{array}$ \\
\hline
\end{tabular}

Sig. vol. - sigmoid volume, T. L. - tandem length

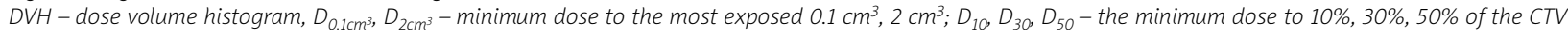
(clinical target volume) 
Increasing bladder volume to about $140 \mathrm{~cm}^{3}$ would cause pushing the applicator posterior towards the rectum and, thus, increasing the rectum dose. However, for patients whose bladder volumes were more than $140 \mathrm{~cm}^{3}$, this effect on rectal dose was negligible. This statement is in agreement with previous research results $[14,16]$. By continuing the bladder distension (more than $140 \mathrm{~cm}^{3}$ ), most of its movement would be upwards (towards the head). It yields a tandem tip orientation, which is more or less horizontal (in the patient supine position), to be directed to the sigmoid and intestine. Comparing the results of Figure 3 and Figure 5, it can confirm this claim. However, these findings are in contrast with some previous studies $[7,24]$, which may be due to the difference in statistical population, dose optimization protocol, and the utilized applicator types.

The dose to the sigmoid can be decreased by filling the bladder up to $75 \mathrm{~cm}^{3}$. For higher bladder volumes, the effect turns opposite again (Figure 5). This can be explained by taking into account the shifting of the bladder due to its various volumes. The discrepancy of the results in this study from those obtained by other authors $[14,24]$ can be explained by the separate delineation of the rectum and sigmoid in the current study. For example, Yamashita et al. defined the rectum and sigmoid colon together as the recto-sigmoid in their study. They found significant reduction of hot spot dose to the small bowel, parallel to the growth of the bladder $\mathrm{D}_{2 \mathrm{~cm}^{3}}$ dose, without any significant outcome in the rectum or sigmoid [24].

The most important factors in choosing the tandem lengths by the physician is the patient's uterine canal length. However, our results showed that the dose to the OARs will be increased by increasing this length. Knowing the relationship of this increase in the dose and the OARs volumes can help the clinicians with choosing the appropriate protocol for the patient preparation before the treatment. An increase in rectum and sigmoid volume has a direct impact on increasing their hot dose points and decreasing their $\mathrm{D}_{10}, \mathrm{D}_{30}$, and $\mathrm{D}_{50}$. Increasing the $\mathrm{D}_{2 \mathrm{~cm}^{3}}$ and $\mathrm{D}_{0.1 \mathrm{~cm}^{3}}$ of these organs will result in telangiectasia, bleeding, and fistula [21,27]. Therefore, controlling these parameters by finding the optimum combination of these organ volumes, bladder volumes, and also tandem lengths can prevent the occurrence of such complications [28].

When the bladder volume was more than $110 \mathrm{~cm}^{3}$, $\mathrm{D}_{0.1 \mathrm{~cm}^{3}}$ and $\mathrm{D}_{2 \mathrm{~cm}^{3}}$ of the sigmoid became constant to some extent and even were decreased for tandem lengths of $\leq 4 \mathrm{~cm}$ (Figure 6). This means that the shifting of the uterus and its consequent effects on the surrounding organ dose is related to the tandem length.

Considering the simultaneous effects of tandem lengths and sigmoid volume, it can be concluded that loading short tandems, a fuller sigmoid will get more dose than an emptier one. On the other hand by inserting a longer tandem, a sigmoid with a lower volume will be exposed to higher doses (Table 3). Therefore, even though the tandem length is not a changeable factor in treatments, the patient's anatomical conditions (i.e., filling status of OARs) can be adjusted to the appropriate length to expose the OARs by a lower dose. For example, the prescription of a two-day bowel preparation before brachytherapy can have a significant impact on the patient's treatment quality.

As mentioned in the above, we used the ANOVA test (GLM) in this study. With knowledge of the assumption of the sample dependence in this test, we were encouraged to perform another statistical analysis by taking into account just one fraction of the data for each patient (i.e., considering only 32 cases) and repeating this test on them. Resulted data for evaluating the bladder dose dependency on its volume, showed a similar trend to the previous results in $\mathrm{D}_{10}, \mathrm{D}_{30}$, and $\mathrm{D}_{50}$ for the bladder. But, for $D_{0.1 \mathrm{~cm}^{3}}$ and $\mathrm{D}_{2 \mathrm{~cm}^{3}}$ of this organ, we were unable to detect any statistically significant differences among different bladder volumes in contrast to our previous results. This might be due to the small statistical population of the new analysis. Furthermore, for the rectum and sigmoid, the trend of this new investigation was the same as the previous one, proving our assumption about the independency of the 97 cases.

In the current research, the patients were not forced to obey any precautions before their therapy, and the effects of bladder, rectum and sigmoid volumes on their received dose were taken into account, in routine treatment conditions. Therefore, we were unable to consider the effects of these organ volumes separately, and consequently some of our results were affected by this fact. For more accurate results, it is recommended that more research is conducted to consider these factors separately by applying specific protocols for patient preparation before the study. The other limitation of this study was that only the physical tandem length could be analyzed. It remains unclear if the main impact is due to the inserted length of the tandem or the length of source loading inside the intrauterine channel.

\section{Conclusions}

There is an optimum volume range for the bladder, rectum, and sigmoid, which can have a direct relationship with the received dose during intracavitary brachytherapy. The effect of a tandem length on the DVH parameters of OARs can be quantified. The most important findings of this study for different organs can be summarized as follows:

Bladder: High-dose parameters of bladder are increase by increasing the bladder volume. Tandems longer than $4 \mathrm{~cm}$ increase the dose to the bladder up to about $4 \%$ (of the planning aim dose) more, compared to the shorter tandems;

Rectum: Rectum dose reaches its minimum and maximum levels for empty and $120-140 \mathrm{~cm}^{3}$ bladders, respectively. Choosing a longer tandem leads to about $5 \%$ more dose to the rectum. For the cases with tandems $>4 \mathrm{~cm}$, $\mathrm{D}_{2 \mathrm{~cm}^{3}}$ of the rectum with bladder volumes more than $80 \mathrm{~cm}^{3}$ is about $10 \%$ (of the planning aim dose) higher compared to the cases with bladder volumes less than $40 \mathrm{~cm}^{3}$. Rectum volume does not have any drastic influence on the dose to rectum for patients whose tandems are $\leq 4 \mathrm{~cm}$.

Sigmoid: When the bladder volume is $70-75 \mathrm{~cm}^{3}$, the sigmoid dose will be minimum. Tandem lengths have a direct relationship with sigmoid dose. By increasing the 
sigmoid volume (i.e., more than $35 \mathrm{~cm}^{3}$ ) the sigmoid dose is increased (e.g. $13 \%$ for $\mathrm{D}_{2 \mathrm{~cm}^{3}}$ ) for the cases who were treated with tandem lengths of $\leq 4 \mathrm{~cm}$. However, the sigmoid dose is decreased when increasing its volume for tandems of $>4 \mathrm{~cm}$.

In conclusion, choosing a bladder with a volume of about $70 \mathrm{~cm}^{3}$ or less is recommended when taking into account the high dose volume parameters for bladder, rectum, and sigmoid.

\section{Disclosure}

Authors report no conflict of interest.

\section{References}

1. Khan FM, Gerbi BJ. Treatment planning in radiation oncology. $3^{\text {rd }}$ ed. Wolters Kluwer/Lippincott Williams \& Wilkins Health, Philadelphia 2012.

2. Viswanathan AN, Thomadsen B; American Brachytherapy Society Cervical Cancer Recommendations Committee; American Brachytherapy Society. American Brachytherapy Society consensus guidelines for locally advanced carcinoma of the cervix. Part I: general principles. Brachytherapy 2012; 11: 33-46.

3. Dankulchai P, Petsuksiri J, Chansilpa Y et al. Image-guided high-dose-rate brachytherapy in inoperable endometrial cancer. Br J Radiol 2014; 87: 20140018.

4. Okuma K, Yamashita H, Kobayashi R et al. A study of highdose-rate intracavitary brachytherapy boost for curative treatment of uterine cervical cancer. J Contemp Brachytherapy 2015; 7: 128-134.

5. Van den Bos W, Beriwal S, Velema L et al. Image guided adaptive brachytherapy for cervical cancer: dose contribution to involved pelvic nodes in two cancer centers. J Contemp Brachytherapy 2014; 6: 21-27.

6. Teke F, Yoney A, Teke M et al. Evaluation of outcome and prognostic factors in 739 patients with uterine cervix carcinoma: a single institution experience. Contemp Oncol (Pozn) 2015; 19: 130-136.

7. Patra NB, Manir KS, Basu S et al. Effect of bladder distension on dosimetry of organs at risk in computer tomography based planning of high-dose-rate intracavitary brachytherapy for cervical cancer. J Contemp Brachytherapy 2013; 5: 3-9.

8. Haie-Meder C, Pötter R, Van Limbergen E et al. Recommendations from Gynaecological (GYN) GEC-ESTRO Working Group (I): concepts and terms in 3D image based 3D treatment planning in cervix cancer brachytherapy with emphasis on MRI assessment of GTV and CTV. Radiother Oncol 2005; 74: 235-245.

9. Pötter R, Haie-Meder C, Van Limbergen E et al. Recommendations from gynaecological (GYN) GEC ESTRO working group (II): concepts and terms in 3D image-based treatment planning in cervix cancer brachytherapy-3D dose volume parameters and aspects of 3D image-based anatomy, radiation physics, radiobiology. Radiother Oncol 2006; 78: 67-77.

10. Lee LJ, Das IJ, Higgins SA et al. American Brachytherapy Society consensus guidelines for locally advanced carcinoma of the cervix. Part III: low-dose-rate and pulsed-dose-rate brachytherapy. Brachytherapy 2012; 11: 53-57.

11. Viswanathan AN, Beriwal S, De Los Santos JF et al. American Brachytherapy Society consensus guidelines for locally advanced carcinoma of the cervix. Part II: high-dose-rate brachytherapy. Brachytherapy 2012; 11: 47-52.

12. ICRU/GEC-ESTRO report no. 89. Prescribing, Recording, and Reporting Brachytherapy for Cancer of the Cervix. Journal of the ICRU [In press].
13. Wyckoff HO, Allisy A, Caswell RS et al. ICRU Report 38: Dose and Volume Specification for Reporting Intracavitary Therapy in Gynecology. International Commission on Radiation Units and Measurements, Maryland 1985.

14. Sun LM, Huang HY, Huang EY et al. A prospective study to assess the bladder distension effects on dosimetry in intracavitary brachytherapy of cervical cancer via computed tomography-assisted techniques. Radiother Oncol 2005; 77: 77-82.

15. Adli M, Garipagaoglu M, Kocak Z. Effect of bladder distention on bladder base dose in gynaecological intracavitary high dose rate brachytherapy. Br J Radiol 2009; 82: 243-248.

16. Kim RY, Shen S, Lin HY et al. Effects of bladder distension on organs at risk in 3D image-based planning of intracavitary brachytherapy for cervical cancer. Int J Radiat Oncol Biol Phys 2010; 76: 485-489.

17. Buchali A, Koswig S, Dinges $S$ et al. Impact of the filling status of the bladder and rectum on their integral dose distribution and the movement of the uterus in the treatment planning of gynaecological cancer. Radiother Oncol 1999; 52: 29-34.

18. Tan PW, Koh VY, Tang JI. Outpatient combined intracavitary and interstitial cervical brachytherapy: barriers and solutions to implementation of a successful programme - a single institutional experience. J Contemp Brachytherapy 2015; 7: 259-263.

19. Pecorelli S. Revised FIGO staging for carcinoma of the vulva, cervix, and endometrium. Int J Gynaecol Obstet 2009; 105: 103-104.

20. Cervix Rotterdam Applicator Set. http://www.elekta.com/ healthcare-professionals/ products/elekta-brachytherapy/ applicators/gyn/Cervix-Rotterdam-applicator.html.

21. Viswanathan AN, SpringerLink (Online service). Gynecologic radiation therapy novel approaches to image-guidance and management. Springer-Verlag, Berlin, Heidelberg 2011.

22. Kirisits C. The EQD2 concept for practical reporting of cervix brachytherapy. www.aoic.net/elekta/elk1402archive/ D201Kirisits.pdf. 2014.

23. Siavashpour Z, Aghamiri MR, Jaberi R et al. A comparison of organs at risk doses in GYN intracavitary brachytherapy for different tandem lengths and bladder volumes. J Appl Clin Med Phys 2015 [In press].

24. Yamashita H, Nakagawa K, Okuma K et al. Correlation between bladder volume and irradiated dose of small bowel in CT-based planning of intracavitary brachytherapy for cervical cancer. Jpn J Clin Oncol 2012; 42: 302-308.

25. Guler OC, Onal C, Acibuci I. Effects of bladder distension on dose distribution of vaginal vault brachytherapy in patients with endometrial cancer. J Contemp Brachytherapy 2015; 6: 371-376.

26. Siavashpour Z, Aghamiri MR, Jaberi R et al. Effect of bladder fullness in brachytherapy procedure for EBRT complete response treated cervical cancer patients. Conference paper. $2^{\text {nd }}$ ESTRO FORUM, Geneva 2013; DOI: 10.1016/S01678140(15)33266-7.

27. Ma JK, Mourad WF, Allbright R et al. Short-term clinical outcome and dosimetric comparison of tandem and ring versus tandem and ovoids intracavitary applicators. J Contemp Brachytherapy 2015; 7: 218-223.

28. Gupta P, Aich RK, Deb AR. Acute complications following intracavitary high-dose-rate brachytherapy in uterine cancer. J Contemp Brachytherapy 2014; 6: 276-281. 